\title{
Developing antiviral surgical gown using nonwoven fabrics for health care sector
}

\author{
*Parthasarathi V, Thilagavathi $\mathrm{G}$
}

Department of Fashion Technology, PSG college of Technology, Peelamedu, Coimbatore - 641 004, India

\begin{abstract}
Background: Healthcare workers' uniforms including surgical gowns are used as barriers to eliminate the risk of infection for both doctor and patient. The prevalence of human immunodeficiency virus, hepatitis B and C viruses in the patient population is very common.

Objectives: To develop antiviral surgical gown comprising of Polypropylene nonwoven as outer layer, Polytetrafluroethylene (PTFE) film as middle layer and polyester nonwoven as inner layer and the surgical gown with a basic weight of $70 \mathrm{~g} / \mathrm{m}^{2}$. Methods: The titanium dioxide $\left(\mathrm{TiO}_{2}\right)$ nano dispersion was prepared with methylene blue and urea as a reacting medium. These nano particles have an average size of $9 \mathrm{~nm}$ which was revealed by High resolution transmission electron microscope. The nonwoven fabric pore size was characterised by using digital image analyzer. The polypropylene nonwoven fabrics were treated with nano dispersion by pad-dry-cure method and trilaminate fabric was formed using fusing machine. The presence of nano particle on the surface of the non woven fabric was confirmed by Scanning Electron microscope.

Results: The trilaminate surgical gown has passed ASTM 1671 viral penetration test which is mandatory for healthcare facilities. The average pore size of inner, middle and outer layer were found as $0.187,0.4$ and 0.147 micron respectively. The tensile strength of the trilaminate fabric in both machine and cross direction was $145 \mathrm{~N}$ and $94 \mathrm{~N}$ respectively. The tearing strength of the trilaminate fabric in direction I and II was $10 \mathrm{~N}$ and $4 \mathrm{~N}$ respectively. The hydrostatic and index puncture resistance of the trilaminate fabric was $2930 \mathrm{mmwc}$ and $58.8 \mathrm{~N}$ respectively. The moisture vapour permeability of the fabric was exhibited as $585.7 \mathrm{~g} / \mathrm{m}^{2} /$ day.

Conclusion: The surgical gown exhibits antiviral property which can protect the health care people from human immunodeficiency virus.
\end{abstract}

Key words: Surgical gown, antiviral gown, Tri laminate, nonwoven, care uniform African Health Sciences 2013; 13(2): 327 - 332 http://dx.doi.org/10.4314/ahs.v13i2.18

\section{Introduction}

The Association of Operating Room Nurses recommended that the fabrics used for gown and drapes must minimize the possibility of passage of bacteria from non-sterile to sterile areas. The surgical gowns should also aid in resistance of liquid transmission, abrasion and punctures. Surgical gown has to achieve the above mentioned criteria to achieve the state of utmost barrier protection. According to these stipulations a surgical gown must meet the following criteria: (a) blood and aqueous fluid resistance, (b) abrasion resistance to eliminate bacterial penetration, and (c) lint free to reduce the number of particles in the air ${ }^{1}$.

\section{*Corresponding author: \\ Parthasarathi Venkatesan \\ Department of Fashion Technology \\ PSG college of Technology \\ Peelamedu, Coimbatore - 641 004, India \\ Email: sarathi_vp@hotmail.com}

The healthcare industry today is very different from what it was 50 years ago. Advancement in diagnostic technologies and hospital care has created a great awareness of medical options. The protective performance of surgical gown fabrics is a major challenge against liquids ${ }^{2}$. The nature of activities in the surgical environment creates an opportunity for gowns to be challenged by body fluids such as blood, perspiration and other liquids such as alcohol or iodine ${ }^{3}$. The necessity of barrier protection and the adherence to federal regulations and professional guidelines make single-use gowns and drapes an ideal choice. Barrier properties are of great importance with the shift from user comfort and cost to user and patient protection. Gowns and drapes act as barriers to prevent transmission of micro-organisms from non-sterile to sterile areas. Impermeability to moisture is a critical factor in choosing materials for gowns and drapes ${ }^{4}$.

Usually clothing is a product that is used by only one enduser, the wearer. However patient gowns are 
unique because they have two types of endusers the patients and the caregivers ${ }^{5}$. The Hospital Association says that while the average visit to a community hospital has become shorter due to an aging population, more and more people are being admitted into hospitals. The cost of providing a modern healthcare system is also increasing year by year ${ }^{6}$. Materials used in surgical gowns should function as protective barriers against the transfer of micro-organisms, particulates and fluids to minimize penetration and the potential for personnel contamination?

Fluid resistance clothing needs to be used, if there is a possibility of splashing or spreading of blood on the fabric. The impact of AIDS and other contagious diseases creates the need of such protective clothing ${ }^{8}$. The protective material also needs to be water proof but breathable to provide comfort to the user'. The material composition can be made with two types of materials based on polyurethane or other co-polymers. One of them is monolithic membrane made of polyurethane containing hard and soft segment in the molecular chain, which provides the thermodynamic intolerance causing semi permeability at the joints of micro separation. The second one is micro porous membrane which allows the body to transmit the perspiration through the fabric ${ }^{10}$. Barrier fabrics are formed by sandwiching nonwoven webs. The melt blown web provides a barrier impervious to bacteria and other contaminants ${ }^{11}$. Surgical drapes are used to protect the patient from infection and also to keep the site of the surgical incision from the risk of infection ${ }^{12}$.

Dane et al found that fabric performance changed after first contact with fluid ${ }^{13}$. Surgical drapes, gowns and gloves are used in the operation theatre to protect the patient and the theatre staff. They also protect theatre equipment from getting damaged. Nonwoven barrier fabrics which are used for surgical gowns and drapes have been developed to impede the passage of bacteria and other contaminants ${ }^{14}$. Association for the Advancement of Medical Instrumentation (AAMI) has suggested standard PB 70:2003 Liquid barrier performance and classification of protective apparel and drapes intended for use in healthcare facilities which is equal to the standard ASTM 1671 intended for healthcare.

\section{Methods}

Non woven fabric is used as a material for developing surgical gown. Three layers of fabrics were used. The outer layer polypropylene nonwoven fabric is used with basic weight of $30 \mathrm{~g} / \mathrm{m}^{2}$ and middle with breathable polytetrafluroethylene (PTFE) film with 0.4 micron with basic weight of $15 \mathrm{~g} / \mathrm{m}^{2}$ and the inner layer as polyester nonwoven fabric with basic weight of $25 \mathrm{~g} / \mathrm{m}^{2}$. Polypropylene is having a moisture regain of $0 \%$ and good liquid repellency. Hence polypropylene is selected as outer layer for the surgical gown. Polyester is having a moisture regain of $0.4 \%$ which helps in absorption of sweat when used for longer duration. Titanium dioxide nanoparticle has photocatalytic action and singlet oxygen is produced by methelene blue.

\section{Preparation of Titanium Nano composite dispersion}

The Titanium tetra chloride ( $\mathrm{TiCl} 4)$ was used as a starting material in this synthesis. $50 \mathrm{ml}$ of $\mathrm{TiCl} 4$ was slowly added to $200 \mathrm{ml}$ of distilled water in an ice cool bath. The beaker was taken from the ice bath to room temperature. The beaker was kept in magnetic stirrer to get a homogeneous solution. Bath temperature was raised to $170^{\circ} \mathrm{C}$ and kept in the same temperature till the process of nano particle formation was completed. In another vessel 30 grams of urea was dissolved in $250 \mathrm{ml}$ of distilled water. From the vessel $160 \mathrm{ml}$ of urea solution was added to beaker under constant stirring. The solution turned into white colloid without any precipitation. 25 grams of Methylene blue was added to the titanium nano particles.

\section{Characterisation of Titanium dioxide Nano composite dispersion}

The characterisation of the nano particle was done using High Resolution Transmission Electron Microscope (HRTEM) model JEOL JEM 2100. The HRTEM confirms the average size of nano particles.

\section{Characterisation of Nonwoven fabric pore size}

A $5 \times 5 \mathrm{~mm}$ swatch was cut from the nonwoven felt. Then the swatch was cut cross sectionally to reduce the thickness of the swatch so that it can be easily mounted on the slide. The surface image of the swatch was taken through the microscope at 400x magnification. Then the area between the entangled fibers was found by software calibrated at $400 \mathrm{x}$ 
magnifications. The average area of 100 readings was determined.

\section{Trilaminate fabric finishing procedure}

The Polypropylene nonwoven fabric was coated with $1 \%$ nano dispersion with material to liquor ratio of $1: 20$ and $0.5 \%$ Anionic binder. The nonwoven fabric was finished with pad-dry-cure method. The excess solution was squeezed using padding mangle which was running at a speed of $10 \mathrm{~m} / \mathrm{min}$ with a pressure of $15 \mathrm{~kg} / \mathrm{cm}^{2}$. After padding, the fabric was dried naturally. The arrangement of three layers of non woven fabric is shown in figure 1. Three layer of fabrics such as nano finished Polypropylene is an outer layer, breathable PTFE film as a middle layer and polyester nonwoven fabric as inner layer were bonded together using a fusing machine at a temperature of $210^{\circ} \mathrm{C}$ with fusing time of $10 \mathrm{~s}$.

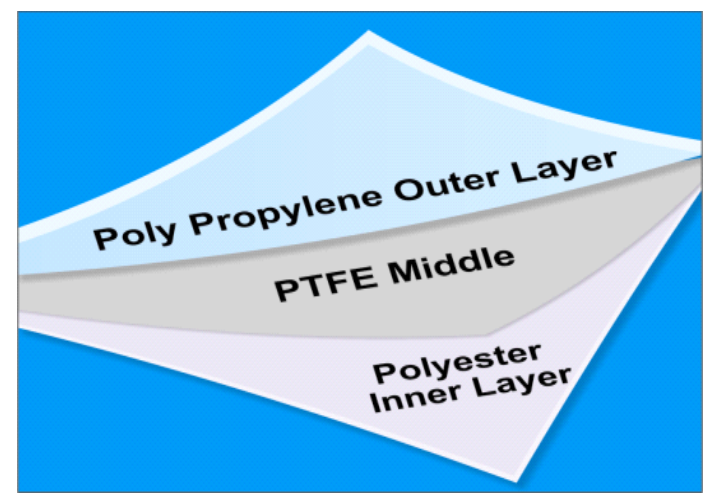

Figure 1: Method of arranging trilaminate fabric

Characterisation of Nano Finished fabric using Scanning Electron Microscope (SEM):

The surface morphology of the nano finished nonwoven fabric was investigated using SEM. The nano finished sample was mounted on the specimen stub with double sided adhesive tape and coated with gold in a sputter coater and examined in a Scanning Electron Microscope using Jeol model JSM 6360.

\section{Evaluation of Trilaminate surgical gown Viral penetration test}

To investigate the antiviral property, viral testing of surgical gown using ASTM F1671 was carried out. ASTM F1671 is the method for testing resistance of materials used in protective clothing for penetration by blood borne pathogens using bacteriophage. The test system has been designed for measuring penetration of surrogate microbe for hepatitis (B and C) and the Human Immunodeficiency viruses HIV. The surrogate Phi$\mathrm{X} 174$ bacteriophage used in the test method is similar to this virus shape and size.

\section{Tensile and tearing strength}

The Tensile strength ASTM D5034 grab test method was used for the determination of breaking force and elongation of nonwoven fabrics. This procedure has been used extensively for acceptance testing in the trade. ASTM D1424 test method is used for the determination of tearing strength by the falling pendulum type apparatus. This test method covers the determination of the force required to propagate a single-rip tear starting from a cut in a fabric and using a falling-pendulum Elmendorf type apparatus.

\section{Hydrostatic resistance test}

The AATCC 127 method was used to measure the resistance of a fabric to the penetration of water under hydrostatic pressure. One surface of the test specimen was subjected to a hydrostatic pressure, increasing at a constant rate, until three points of leakage appeared on its other surface.

\section{Index puncture resistance test}

ASTM D4833 was used to measure the resistance of nonwoven fabric and stimulate the puncture strength of the fabric to static loads of aggregate. In this test, the sample was secured in a ring clamp. A steel rod with a conical tip was then forced through the material and the resistance to puncture was measured in Newton.

\section{Moisture vapour permeability test}

The Moisture Vapour Transmission test was conducted according to ASTM E96 standard. The cup method was used with the air at relative humidity of $50 \%$ and recommended water temperature of $32.2^{\circ} \mathrm{C}$. The moisture vapour permeability was measured in terms of $\mathrm{g} / \mathrm{m}^{2} /$ day.

\section{Results}

Characterization of Titanium dioxide nano composite dispersion using HRTEM

The morphology of $\mathrm{TiO} 2$ nanoparticle was revealed using HRTEM image shown in figure 2 . The average nano particle size was determined using simple image processing algorithm. The average diameter of the nano particle is $9 \mathrm{~nm}$. 


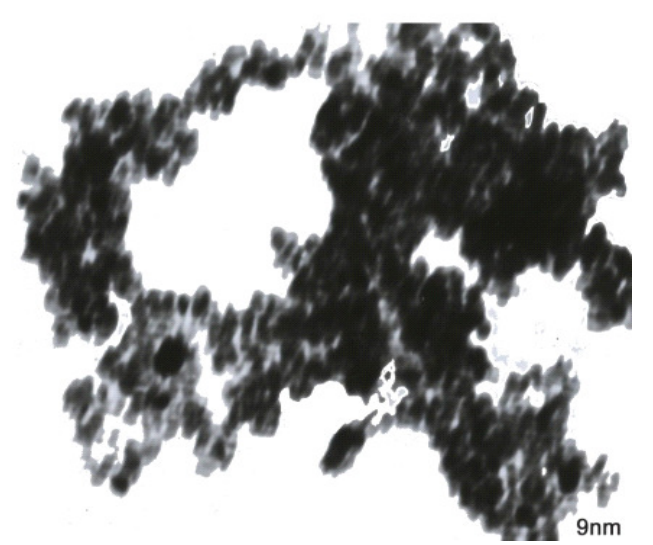

Figure 2: TEM image of $\mathrm{TiO}_{2} \mathrm{Nano}^{\mathrm{T}}$ article

Characterisation of nano finished fabric using SEM

The surfaces of nano treated nonwoven fabrics were observed by SEM. Figure 3 shows the image of untreated fabric which shows clear rod like appearance. The nanoparticles are well dispersed on the fibre surface as shown in figure 4 . The nano particle agglomerates are seen on the surface fibre. By using padding mangle for application of nano particle on the nonwoven fabric, the nano particle penetrates into the fabric matrix.

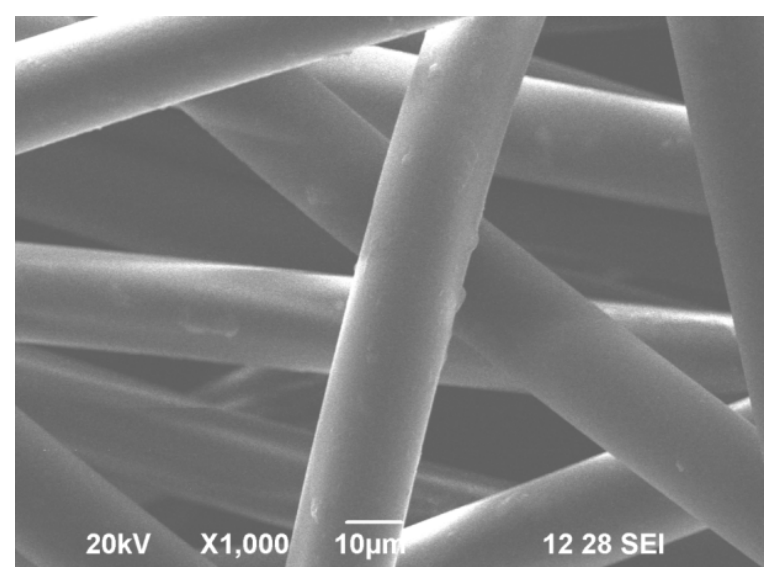

Figure 3: SEM image of untreated nonwoven fabric

Characterization of nonwoven fabric pore size

The average pore size of Polypropylene nonwoven fabric, Polytetrafluroethylene film and Polyester nonwoven fabric was found as $0.187,0.4$ and 0.147 micron respectively. The Outer layer polypropylene nonwoven fabric with smaller pore size increases the protection level. The middle layer film provides breathability helping wearer to wear the gown for longer duration

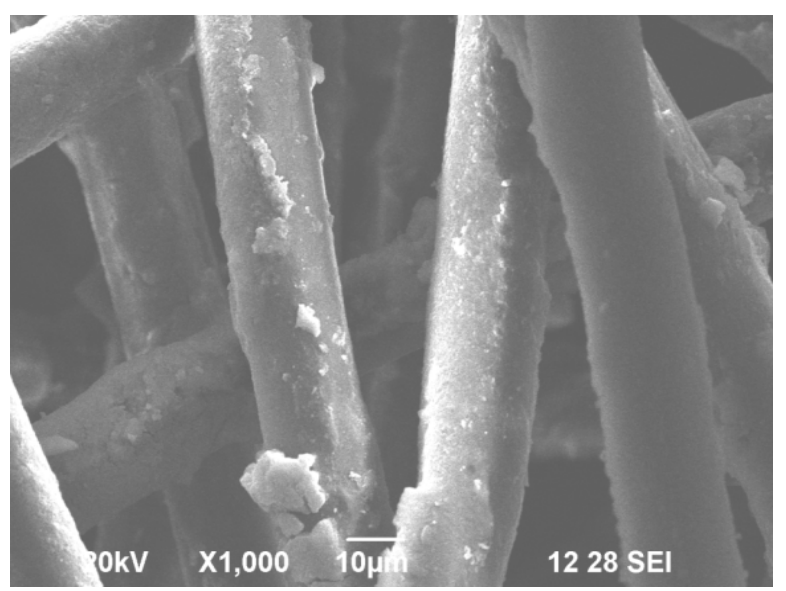

Figure 4: SEM image of nano treated nonwoven fabric

\section{Evaluation of trilaminate surgical gown}

The table 1 shows the result of various evaluations conducted on the single layer and trilaminate surgical gown. The trilaminate fabric has passed the viral penetration test, which confirms that the gown has protection against the hepatitis (B and C), Human Immunodeficiency Viruses and surrogate Phi-X174 bacteriophage. The single layer gown does not provide protection against viruses due to the absence of polytetrafluroethylene film and inner layer of polyester nonwoven fabric. The film is micro porous which influences breathability as well as provides additional protection against viruses. In this research work polypropylene outer layer has a pore size of 0.187 micron which is mainly considered for protection. The tensile strength of the fabric in machine direction is $145 \mathrm{~N}$ which is greater than the cross direction of the sample which is $94 \mathrm{~N}$. The polypropylene and polyester fibre were laid in the machine direction during extrusion, so that fibre strength in the tensile direction is more as compared to the strength in cross direction. The tearing strength of the fabric in the direction I was more due to same reason as quoted above. The hydrostatic resistance test reports show the result as $2930 \mathrm{mmwc}$ which confirms the grade Level 2 protection. The index puncture resistance of the fabric was found as $58.8 \mathrm{~N}$. The moisture vapour permeability tests exhibited result of $585.7 \mathrm{~g} / \mathrm{m} 2 /$ day which also confirms the gown requirements. The gown has also good moisture vapour permeability so that the health care people can wear the gown for long duration with comfort. 
Table 1: Results of various parameters of single layer versus Trilaminate surgical gown

\begin{tabular}{|c|c|c|c|}
\hline Parameter (Unit) & Standard & Single layer gown & Trilaminate gown \\
\hline Viral penetration test & ASTM F 1671 & Fail & Pass \\
\hline Tensile strength $(\mathrm{N})$ & ASTM D 5034: 2009 & & \\
\hline Machine direction & & 130 & 145 \\
\hline Cross direction & (Grab) & 86 & 94 \\
\hline Tearing strength & & 7 & 10 \\
\hline Direction I (Cross wise tear) & ASTM D 1424:2009 & & \\
\hline Direction II & & 2 & 4 \\
\hline Hydrostatic resistance (mmwc) & AATCC 127:2003 & 1850 & 2930 \\
\hline Index puncture resistance $(\mathrm{N})$ & ASTM D 4833 & 46.5 & 58.8 \\
\hline $\begin{array}{l}\text { Moisture vapour permeability } \\
\left(\mathrm{g} / \mathrm{m}^{2} / \text { day }\right)\end{array}$ & ASTM E 96 & 634.3 & 585.7 \\
\hline
\end{tabular}

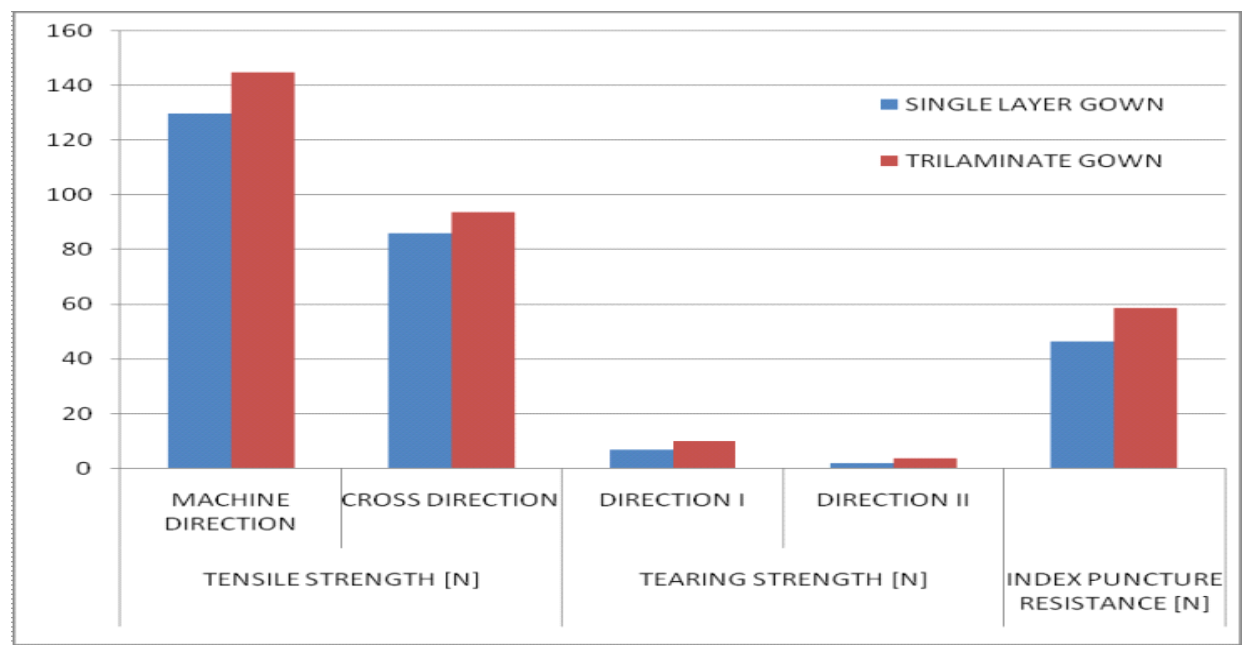

Figure 5: Results of Tensile, tearing and index puncture resistance of Single layer Vs trilaminate gown

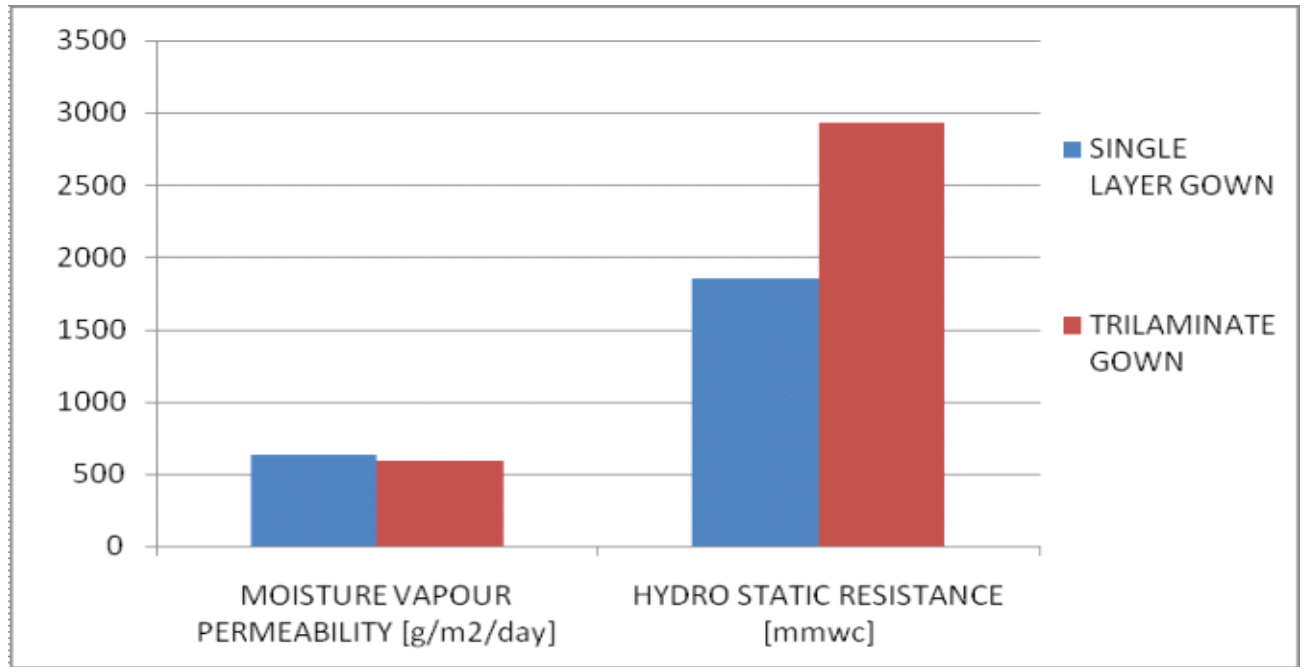

Figure Figure 6: Results of moisture vapour permeability and hydrostatic resistance of Single layer Vs trilaminate gown 


\section{Discussion}

There has been always an endeavour to impart viral barrier properties in fabrics used for surgical gown so that the protection gets improved. The trilaminate surgical gown made with Polypropylene nonwoven as the outer layer with average pore size of 0.187 micron thereby provides Level 4 protection. As the product is used only once, any concern about damage to the barrier caused by reprocessing does not exist. Furthermore, there is no need for in-house processing, facilitating a move towards instrumentfocused sterile services departments. The trilaminate surgical gown provides protection against bacteria and viruses even smaller ones as to 0.027 micron. Liquid barrier property is also established in this surgical gown thereby reducing cross-contamination between medical staff and patient's body fluids. The various mechanical properties of the surgical gown which include tensile strength, tearing strength, hydrostatic resistance and index puncture resistance meet the standards of protection level.

As the surgical gown is of light weight and comprised of breathable fabric it confirms the user of the product that it is comfortable to use. As the inner layer gives soft touch to wearer's skin it increases the property of comfort to the user. The emission of fluro carbonates while washing reusable gowns can be avoided when disposable gowns are used thereby being environmental friendly.

Only $2 \%$ hospital waste is contributed by disposable gowns. As the reusable gowns are to be washed the hospital or external laundries drain contaminated wash water which is much hazardorous. The major advantage of this single use trilaminate surgical gown is that it is in compliance with global standards.

\section{Conclusion}

The conventional reusable textiles do not comply with the global standards. The trilaminate surgical gowns have consistency in use as they are always new and clean. Hence there is no need of Compromise during laundering.

\section{References}

1. Association Operating Room Nurses. Recommended practices: Universal precautions in the preoperative setting. Association of Operating Room Nurses (AORN) Journal 1993;57: 554-558.

2. Azouni MA, Petre G. Surface-tension-driven flows in a thin layer of a water solution. $J$ of Colloid and Interface Science 2001; 239: 509-516.
3. Average Length of Stay in Community Hospitals (2011). Retrieved from http://www.aha.org/ aha/trendwatch/chartbook. (accessed 24 october 2011)

4. Barbara J. Single use Vs reusable gowns and drapes. Infection control today 2002;1: $\quad 3234-$ 37.

5. Belkin $\mathrm{N}$. The challenge of defining the effectiveness of protective aseptic barrier. Technical Textiles International 1993;1: 22-4.

6. Federal Register. Occupational Exposure to Blood borne Pathogens: Proposed Rule and Notice of Hearing. Part II, Department of Labour. Occupational Safety and Health Administration 1995; 5: 23042-139.

7. Lickfield. Nonwovens in medical textiles. International fibre J 2001; 3: 42-8.

8. McCullough EA, Schoenberger LK. Liquid barrier properties of nine surgical gown fabrics. $J$ of Nonwoven Research 1993;4: 14-20.

9. McCullough EA. Methods for determining the barrier efficacy of surgical gowns. American J of Infection Control 1995;21: 368-74.

10. Phongikaroon S, Hoffmaster R. Effect of temperature on the surface tension of soluble and insoluble surfactants of hydro dynamical importance. $J$ of Chemical and Engineering Data 2005;50: 1602-7.

11. Rigby AJ. Medical Textiles; Textile Materials in Medicine and Surgery. Textile Horizons 1993; 2: 1216.

12. Rutala WA, Weber D. A review of single-use and reusable gowns and drapes in health care. Infection Control and Hospital Epidemiology 2001; 4: 248-257.

13. Unsal E, Dane JH, Schwartz. Effect of liquid characteristics on the wetting, capillary migration, and retention properties of fibrous polymer networks. J of App Polymer Sci 2005; 97: 282-92.

14.Zhang X, Raheel M. Statistical model for predicting pesticide penetration in woven fabrics used for chemical protective clothing. Bulletin of Environmental Contamination and Toxicology 2003;70: 652-9. 